frais particuliers pour ladite Administration ou Compagnie et à ce que soient prises toutes mesures de précaution et de sécurité nécessaires, solt pour tout ou partie, par ladite Administration ou Compagnie elle-même, si elle le demande et que l'arrêté préfectoral le stipule.

Le coût des travaux exécutés par ladite Administration ou Compagnie pour le compte du permissionnaire, annsi que tous frais de gardiennage, 'éclairage, surveillance, vérification des installations, main-d'œuvre, fournitures, etc., exposés par elle à l'occasion de l'établissement, de l'entretien et de la réparation desdites installations, lui seront remboursés par le permissionnaire dans le mors de leur achèvement, avec une majoration de $15 \%$ pour frais généraux et intérêts.

$$
\text { ART. } 21 .
$$

Le permissionnaire devra, chaque fois quil en sera requis, solt par le service du contrôle, soit par l'Administration exploitante ou la Compagnie concessionnaire du chemin de fer, mettre gratuitement à leur disposition tous instruments de mesure et autres moyens nécessaires pour la vérification - aux frais dudit permissionnarre, au cours de l'exploitation du réseau électrique dont feront partie les conducteurs traversant le chemin de fer - de la tension ct de l'intensité du courant dans ces conducteurs et du bon isolement desdits sonducteurs par rapport aux masses métalliques de la voie ferréc et des lignes électriques dépendant du chemin de fer

$\mathrm{Ce}$ permissionnaire sera tenu, en outre, de communıquer régulièrement auxdits services du contrôle et Administration ou Compagnie les procès-verbaux des mesures d'isolement exécutées sur ledit réseau électrique.

Les vérifications prévues au premier alınéa du présent article seront effectuées, les unes pérodiquement, à raison d'une par année, les autres inopinément Ces vérifications seront effectuées en présence du permissionnaire ou lui dûment convoqué, et, si le service du contrôle y est représenté, donneront lieu à un procès-verbal de ce service.

ART. 22.

Avant la mise en service des conducteurs électriques, les installa. tions autorisées seront récolées par un agent du service du contrôle pourvu hiérarchiquement de l'arrêté d'autorisation, en présence ou après due convocation du service départemental des postes et télégraphes, de l'Administration exploitante ou Compagnie concessionnaire du chemin de fer, et dı permissionnaire.

Paris, le $\mathrm{I}^{\text {er }}$ février 1907 .

$$
\begin{gathered}
\text { Le Ministre des Travaux publics, } \\
\text { des Postes et des Télégraphes, } \\
\text { Louis BarTHou. }
\end{gathered}
$$

\section{Circulaire du Ministère de l'Agriculture, du Jer juin 1906, relative au nouveau règlement de police des cours d'eau non navigables ni flottables.}

Par une circulaire du 17 juillet 1900, les Préfets ont été invités à rapporter le règlement de police sur' les cours d'eau non navigables ni floltables qu'lls avaient dù prendre conformément aux instruction de M. le Ministre des Travaux publics en date du 21 juin 1878.

Cette mesure était indispensable puisque le modèle réglementaire n'étast plus en parfaite concordance avec la loi du 8 avril 1898 sur le régime des eaux ; mais l'absence de tout règlement de police ne pouvait être que provisoire, étant donnée la nécessité, pour l'Administration, de fixer avec précision les usagers deis eaux sur' leurs obligations, et un nouvcau règlement devait être pris par les soins des Préfets, dès que la loi du 8 avril 1898 aurajt été complétée par les règlements d'Adminilstration publıque prévus par" son application.

Les formalités relatives à l'instruction des demandes concernant les ouvrages soumis à autorisation venant d'être déterminées par le décret du $1^{\text {er }}$ août 1905, les préfets doivent prendre, dans le plus bref délai possible, un arrêté portant règlement de police sur les cour's d'elau non navigables ni flottables de leur département conforme au modèle annexé à la présente circulairre $\left(^{*}\right)$.

(*) On trouvera la teneur de ce décret dans le numéro de février 1906 de la La Houille Blanche, page 44.
Plusieurs Préfets ayant, à diver'ses reprises, demàlt des instructions sur l'étendue des pouvoirs de l'Admunistration en matière de police des eaux, la présente curculaire donnora ciraprès, sur la portée des divers articles du règlement, quelques indications sur lesquelles l'Admmistration appelle toute leur attention, ainsi que celle des agents du service hydraulique.

Article premien. - Recépage des arbres. - Liarticle $1^{\mathrm{er}}$ quı prescrit aux riverains le recépage des arbres, buissons et souches formant sallie ne souleve aucune obscrvation particulière. Il convient, cependant, de signaler que l'obligation permanente qu'il leur impose est absolument indépendante de celles quil pourraient leur incomber en ce qui concerne les opérations spéciales de curage et de faucarde. ment qui seraient ordonnées dans les conditions prốvues au chapilie 3 du titre II de la loi sur le régime des eaux.

AnT. 2 et 3. - Produits des curages et passage sur les mopriétés riveraines. - Les articles 2 et 3 assujehlissent les riverains à recevoil' sur leur's terrams los matières provenant des curages ot à livrer passage aux agents de l'Administralion préporsés à la surveillance des cours d'eau, ainsi qu'aux entrepreneurs el ouvriers chargés du curage.

Ces servitudes qui frappent les propriétés riveraines, déri. vent de la situalion mème des lieux et sont consacrés pro la jurisprudenice.

AnT. 4. - Caractères distinctifs des travaux subordonnés d une autorisation préalable. - Larticle 4 a pour bul diñ̂tiquer de la façon la plus gténérale quels sont les fra. vaux dont l'exécution est subordonnée à une autorisalto' préalable.

Toutes les fois qu'un travail quelconque permanent ou temporaire, est susceptible d'avoir une influence, soit sur le régime, solt sur l'écoulement des eaux, ll ne doit êtro ontrepris qu'après avoir été auparavant autorisé par l'Administration.

Pour reconnaître, dans chaque espèce particulière, si une autorisation est nécessaire, il conviendra donc uniquement d'examiner si l'une ou l'autre de ces deux conditions est remplic. Cette règle est absolua ; elle est applicable quelles que soient la nature et l'importance de l'ouvrage projeté, quelle que sioit sa situation par rapport au lil. Elle conserve toute sa valeur lorsque l'opération ne comporte pas d'ouvrages intéressants par cux-mèmes l'écoulement des caux, comme par exemple dans le cas de dárlivátions effectuées au moyen de simples coupures dans la berge. Elle trouverast également son application' s'il s'agissait de prises ou déversemenls communiquant indirectement avec une rivièr, puisque, danis ces circonstances, le régime des eaux serait encore influencé.

Les caractères distinctifs des travaux soumis à autorisation étant aínsi délorminés par l'article 4, les arliclos 5, 6, 7 et 8 envisagent les différents cas qui peuvent se présenter et préciscnt los règles spéciales à chacun d'eux.

ArT. 5. - Travaux dans le lil des cours d'eau. - Larticle 5 vise les travaux dans le lit qui intéressent nécessailrement l'écoulement des eaux, el qui, à ce titre, ne peuvent être exécutés qu'après avoir été autorisés, qu'il siagisse do barrage, d'épis ou même de simples terrassenents ou de plantations. D'après la loi du 8 avril 1898, le préfet est compétent pour accor'der less autorisations nécessaires, après l'accomplissement des formalités prescrites par le décrét du $f^{\text {er }}$ août 1905 .

Il conviendra, d'ailleurs, de faire encore l'application de l'article 5 dans le cas de travaux quil, sans être complètement dans le lit empieteraient sur les limites naturelles du cours r'eau.

Lorsqu'il ne s'agira que de réparations à des ouvrages précédemment autorisés, pour ne pas multiplier, sans nécessité, les sujétions imposées aux riverains et pour réduire autant que possible le nombre des affaires à instruire par 
les agents du service hydraulique, il n'a pas paru indispensable d'exiger une autorisation. Mais, ll doit être bilen entendu que si les réparations ne devalent pas laisser les ouvrages identiques à ceux précédemment autorısés, sill s'agissait non de réparations proprement dites, mais de modifications, une autorisatıon serait nécessaire. Elle le serait égaloment même dans le cas de simples réparations, si les ouvrages remı en état n'avaient pas été précédemment autorisés ct ll conviendralt alors de procéder à la réglementalıon de l'ensemble des ouvrages non autorisés.

ART. 6. - Extraction par les riverains dans le lit. - L'article 6 apporte une nouvelle exception au principe qui subordonne à une autorisation préalable l'exécution d'un travail quelconque dans le lit des cours d'eau. Celte tolérance concerne lesextractionsde vases, de sable et de pierres par les riverains.

Antérıeurement à la low sur le régıme des eaux, le lıt des cours d'eau non navigables ni flottables élant " res nullius", l'autorisation d'extraire des matériaux a été fréquemment accordée par l'Administratıon à des non riverains; aujourd'hui, cetle pratique n'est plus possible, puisque la loi a reconnu aux riverams la propriété du lit et leur a attribué comme conséquence le droit exclusif d'extraire la vasie, le sable et les pierres, à leur profit. Mais, dans l'exercice de leurs droits de toutes nalures, les riverains sont soumis au pouvoir réglementaire de l'Administiatıon, et l'artıcle 3 de la loi du 8 avril 1898 prévoit de plus, expressément, que les travaux d'extraction ne dolvent pas mordifier le regime des eaux. On pouvant se demander sl, dans ces conditions, ll n'y avail pas lieu d'assimiler" ceux-ci' purement et simplement aux autres terrassements dans le lit et de les subordonner, par suite, à une autorisatıon individuelle préalable.

Il a paru que cette obligation serait, dans une certaine mesure, gênante pour les intéressés et qu'elle n'était pas indispensable, étanl donné, d'une part, que les extractions ne peuvent en général apporter de troubles sérieux au régime desi cours d'eau ; d'autre part, qu'il est toujours possible au Préfet de réglementer ou méme d'interdire complètement celles qui présentieranent des inconvénilents pour l'intérêt geénéral, au point de vue de l'écoulement des eaux comme au point de vue de la salubrité.

Cependant, il y aura le plus souvent intérêt à ne pas lassser aux riverains une liberté absiolue en ce qui concerme l'extraction de la vase, du sable et des pierres, et il apparliendra au Préfet, ainsi que le prévoit l'artucle 6, de fixer les conditions générales auxquelles sera sioumis l'exercice de ce droit en vue d'assurer le libre écoulement des eaux, de sauvegarder la salubrulé et de préserver les ouvrages publics tels que ponts, digues, travaux de défense ou d'alimentation de canaux, ctc., établis par l'Etat, les départements, les communes ou par les associations syndicales.

Les prescriptions qu'il conviendra d'édicter pourront d'alleurs ne pas être iden.lilques pour tous les cours d'eau du département, étanl donnée la diversité des naturesı de leurs lits. Après avoir demandé, au sujet des mesures à prendre, des propositions aux Ingénieurs du service hydrau. lique, les Préfets soumettront à l'examen du Ministre de l'Agriculture leur arrêté réglemenlaire avant de le porter à la connaissance du public.

Ant. 7. - Ouvrages au-dessus des cour's d'eau ou les joignant. - Liarticle 7 concerne les ouvrages au-dessus des cơurs d'eau ou les joignant et prescrit, pour leur' établisse. ment, des formalités que l'Administration a élé amenée par l'expérience à adopter, tant dans l'intérêt général que dans celui des riverains.

En principe, il avait semblé posisible de laisser les intéressés exécuter librement, à leurs risques et périls, les ouvrages de cette nature, sauf à ordonner la démolition de ceux d'entre eux qui, préjudiciant à l'écoulement des eaux, sont interdits par l'article 10 de la loi du 8 avril 1898. Mais l'application de cette procédure à fait ressortir de tels inconvé- nients que le Minıstre a dû abroger la circulaire du 17 juillet 1900 qui l'avait instituée, en ce qui concerne les ouvrages joignant le lit.

Les riverains se rendent, en effet, difficilement compte de l'influence que peuvent avoir sur" le rég"imel des eaux les travaux qu'lls se proposent d'exécuter et, comme ils ont une tendance naturelle à avancer autant que possible leurs constructions vers le lit, le nombre de celles d'entre elles qui gênent l'écoulement augmente tous les jour's. Dans ces conditions, l'Administralion ne pourrait continuer à user de tolérance, sans compromettre l'intérêt général, et elleı seraat avant peu dans l'obligation de pour"suivre la démolition de tous les ouvrages faisant obstacle au libre écoulement des eaux. De semblables mesures de rigueur ne pourraient manquer d'être très préiudiciables aux intéressés et seraıent. d'autant plus regrettables que la plupart d'entr'e eux n'ont établ qué par ignorance des ouvrages nuisibles. Ces.ind1cations montrent bien que, dans l'inlérêt mème des riveraıns, ll est préférable de ne pas maintemir un régime de liberté absolue qui, sans leur donner" aucun avantage réel, peut entraîner" de sérıeuses difficultés ultérieures, et que l'exécution de travaux poignant le lit ou au-dessus doit nécessaurement être subordonnée à certaines formalités.

L'Administration ne pouvalt songer à fixer', d'une manière générale, les conditions d'établissement des ouvrages de cette catégorie, car', sur" un mêmei cour's d'eau, les variatıons de régime sont fréquentes et la détermination de ces condltions exigerast par" suiter un travail aussı long que difficile.

Le procédure fixée par l'artıcle 7 qui oblige les riverains à soumeltre au Préfel, avant leur exécutıon, lesı dispositıons qu'lls se proposent d'adopter était dona la seule pratique : les sujétions qu'elle improse aux intéressés sont très faibles par rapport à la sécur'té qu'elle leur donne, et l'on ne peut trouver de meilleures preuves de ses avantages que dans le fait qu'actuellement un grand nombre de riverains communiquent, de leurr pleın gré, leurs projets à l'Administration, avant de les metlre à Exécution, dans le but de savoirt s'lls ne nuisent pas à l'écoulement des eaux.

Les formalités prescrites par l'article 7 ne soulèvent aucune observation spéciale ; il convent cependant de remarquer que le délai de deux mors qui est imparti au préfet pour faire connaitre aux pétitionnaires si l'ouvrage projeté intéresse ou non l'écoulement des eaux ne s'applique évidemment pas à l'arrêté préfectoral à prendre, si l'ouvrage doit faire l'objet d'une autorisation, arrêté quı sera rendu après enquête dans les conditions prévues par le décret du $1^{\text {er }}$ août 1905.

Art. 8. - Prises et déver'sements d'eau. - Lartide 8 est relatif aux prises et déversements d'eau.

LES riverains dans l'exercice des clroits sur les eaux qui leur sont conférés par le code civil sont soumis au pouvoir réglementaire de l'Administration, el l'article 2 de la loi du 8 avrıl 1898 rappelle expressément que, dans l'uslage de l'éau courante, qui borde ou traverse leurs propriétés, ils sont tenus de se conformer aux dispositıomis des règlements el aux autorisations émanées de l'Administration.

Les pirises et les déversements d'eau nut nécessarrement, quel que sont le volume dérivé ou écoulé, une influence sur le régime du cours d'eau où ils sont effectués, et devraient, par suite, confor'mément au principe posé à l'article 4, n'être opérés qu'après avoir été autorisés. Cependant l'application stricte de celte règle entrainerait un travail considérable pour les agents du service hydraulique, imposerait dans certains cas aux riverains des sujétions sans utilité ; il convient de ne pas exiger d'autorisation toutes les fois que le débit du cour's d'eau ne serait pas modifié d'une manière appréciable.

Dansi ce cas, en effet, une réglementation n'est pas utile pour les, déversements qui n€ peuvent présenter" aucun inconvénient et elle n'a également aucune raison d'être pour les priises, puisqu'étant donné leur faıble importance, elles 
ne peuvent priver les usages d'aval de l'eau qui leur est indispensable.

Dans le cas contraire, une autorisation préalable devra être demandée: pour toutes les prises ou déversemen:s envisag'és au seul point de vue du volumie dérivé ou écoulé et indépendamment des ouvrag es destinés à les effectuer, soumis par èux-même à la réglementation s'ils intéressent l'écoulement des eaux. Cetter autorisation sera d'ailleurs nécessaire, que ces prises ou ces déversements soient faits directement ou indirectement dans les cours d'elau, et même s'ils ne devaient être' que temporaires.

Il conviendra, en particulier, de n'apporter aucune tolérance à cet égard loules les fons qu'ıl s'agira de dérivations pouvant nuire à la salubrité, à l'alimentationı des hommes ou des animaux, aux bessoins domestrques, à l'utilisation générale des eaux ; dans ces cas, l'intervention de l'Administration devra nécessairement s'exer"cer" pour sauvegar"der ces intérêts dont elle dont tout parliculièrement se préoccuper ainsi qu'il șera indilqué avec plus de détaıls à propos de l'article 11.

L'autorisation deva être précédée des formalıtés prévues par le décret du $1^{\text {er }}$ août 1905 et la circulairer dont est annoncé l'envoi, qui précisera les conditions d'applicalion de ce décret, donnera des instructions complètes, en, ce quı concerne les dispositıons à insérer dans les règlements individuels.

Il y a lieu d'appeler tout spécialement l'altention, d'une part, sur' ce que les non-riverains, sauf ceux auxquels des titres auraient par" exception conféré des droits sur les eaux, ne peuvent pratıquer de dérivationsi sur les rivières non navigables ni flottables qu'en vertu d'une déclaration d'utilité publique ; d'autrie part, sur ce que les déversements susceplibles d'être autorisés par" appilication de l'article' 8, ne peuvent évidemment être que des déversements d'eaux propres, ne rentrant pas dans la catégorie der ceux qui sont interdıts par l'article 12.

ART. 9. - Obligations des usiniers relatives à l'écoulement des eaux. - L'article 9 est relatif aux obligations des usiniers et-des usagers des barrages, en ce qui concerne l'écoulement des eaux.

Les trois premiers paragraphes s'appliquent à toutes les usines, qu'elles sorent réglementées ou non. Le premier interdit de placer aucune hausse sur les déversonrs et les vannes des usines de façon à ce que les usiniers ne puissent surélever, à l'insu de l'Administration, le niveau de leurs retenues et nure ainsi à l'utilisahon agricole ou industrielle des eaux, soit en augmentant l'humidité des lerres, soit en allongeant la durée des intermittences sur les rivières où les usimes par éclusées.

Le deuxième indique que les usiniors sont responsables de la surélévation des eaux, tant que les vannes de décharge ne sont pas levées à toute hauteur.

Le troislème concerne les lâchur'es et l'entretien des ouvrages sujets à la réglementation.

La clause r"clalive aux lâchures. s'applique plus spécialement aux usines comportanl des relenues qui emmagasinent un volume considérable; dans ce cas, il importe de ne jamais fair"e la vidange de ces retenues qu'en prenant toutes les précautrons nécessaires pour éviter une inondation à l'aval.

L'obligation d'entretenir les ouvrages vise tout particulierement les bar"rages réservoirs. Il ne suffit pas, en effet, que les arrêtés réglemenlaires des usines déterminent les profils en travers de ces barrages en tenant compte de leur morde de constructıon de façon que leur stabilité soit assurée, il faut encore que leur entreben soit constant, pour" qu'une rupture, qui entrầnerait la ruine de la vallée à l'aval, ne puisse se produir"e.

Les deux derniers paragraphes concernent les usines non pourvues de titres réglementaires.

Le quatrième parag'aphe stipule qu'à défaut de titre r'é- glementaire, les eaux ne devront pas dépasser le dessus du réservoir ou de la vanne de décharge la moins élevée s'il n'existe pas de réservoir. Cette prescription a pour but de prévenar" les inondations, en obligeant les usagers des retenues non réglementées à établir leurs ouvrages de façon que les eaux puissent s'écouler comme si l'usine n'exıstait pas. Les barrages non autorisés ne peuvent être tolérés, lorsqu'ils ne r'emplissent pas les conditions pr'écédentes, que s'ils sont construits de façon à être emportés pour la première crue.

Le cinquième paragraphe de l'article 9 indique que les usiniers ét usagers des usines réglcmentées sont responsables de la surélévation des eaux, soit qu'elle provienne du défaut de manœuvre de leurs vannes, solt qu'elle résulte de la trop grande hauteur du déver"soir" ou de l'insuffisance des ouvrages de décharge.

S'il s'agil d'usines réglementées, établies, conformément à leur acle d'autorisation, la r'esponsabilité pénale n'existe que lorsque la manœuvire des-vannes n'a pas été faile en temps utile, of l'usmier ne pourrait êtro, cn cas de dommages résultant de dispositions défeclucuses des ouvrages fixés par liddministration, que rondu civilement responsable vis à-vis des hers dont les droits demeurent toujours réservés.

AnT. 10. - Obligations des usiniers et usagers des barrages pendant les opérations de curage. - Larlicle 10 prescr"t aux usiniers et usag'r's des barrages l'obligation do tenir leurs vannes ouvertes pour l'exécution et la réception des trqvaux de curage aux jours fl heures fixés par les arrêlés préfcctoraux. Les chômages qui leur sont ainsi imposés sont largement justifiés, d'une part, parce que les curag'os leur profitent directement $\in \mathrm{n}$ rétablissant le cours régulicr des caux ; d'aulre part, par'ce que l'encombrement du lit par les dépôts est en grande partie la conséquence du rcmous créé par les ouvrages de relenue.

ART. 11. - Transmission des eaux. - D'une manière générale, les dispositıons des artıcles précédents, șauf celui qui concerne les prises, ont pour objet de plévomir les inondations ; mais l'Admmistrabion n'a pas à ser préoccuper seulement d'assurer aux eaux leur libre écoulemenl, elle doit aussi sauvegarder les autres inlérêts généraux qui lui sont conflés, notamment la salubr'té el l'alimentation des populatrons ; elle doit encore intervenir prour" que les eaux reçouvent la molleure utılisation possible, pour les diriger' comme le prévolt la lo1 des 12-20 aonll 1790, vers un bul d'utilité générale.

L'articlo 11 impose, à cct cffot, aux usiniers el aux usagers des prises indépendammont des obligations yui peuvent résulter pour eux des règlements gênéraux do répartition des caux entre l'Agriculture et l'Industrie ob de leur's règlements d'eau particuliers, l'observalion de diverses prescriptions relativement à la transmission des eaux.

Le premier paragraphe de l'article 11 est aesting à protéger la salubrilé publique, à assurer aux populations d'aval l'eau nécessaire à leur alimentaion ainsi qu'à l'abreuvement de leur's bestiaux et à la satisfaction de leurs besoins domes. tiques. La dactrino et la jurisprudence sont d'accond pour reconnaître la légıtimité de l'intervention de l'Adminishra. tion en faveur de ces intérêts généraux dont la protection s'impose d'autant plus que les besorns de celle nature ne sont pas garantis d'une manière expresse par le Code civil.

Il doit être bien entendu, d'ailleurs, que l'obligation générale ainsi édictée ne doit pas empêcher le Préfet de prendre, dans les qèglements d'eau individuels, en vue de sauvegarder ces mêmes intérêts, des mesures spéciales et précises, qui sont en particulier presque toujours indispensables dans les cas de prises importantes ou d'usines établies sur des cours d'eau à faible débit où la martche par éclusées est normale.

Les prescriptions du premier paragraphe de l'article 11 ne suffisent pas a sauvegarder les intérêts généraux de toute 
nature dont l'Administration a la garde, lorsqu'il s'agit d'usines dont les retenues s'étendent sur de très grandes longueurs ou qui emmagasment un volume d'eau considérable, soit au moyen de barrages réservoirs construits sur les cours d'eau, soit par des réservorr's exécutés en dehors du lit, mais alimentés par une dérivation.

Les usines de cette imporlance apportent dos porturbations profondes au régime des riviores sur lesquelles elles sont établies, puisqu'elles peuvent interrompre le cours naturel des eaux sur plusieurs kllomètres et exercer leur action jusqu'à une distance encore plus considérable à l'aval do lour canal de fuite en retenant les eaux, ou en ne les transmettant que d'une manuère intermiltente.

Dans ce cas, l'Administration, ne peut par sulte, borner son rôle à prescrure l'écoulement du débit nécessasrc à la salubrité of à l'alimentation publiques, ello dont encore prendre les mesures indıspensables pour que les ruverains et les usagers d'aval ne solent pas mis dans l'mmpossibilité d'exercer" leurs drouts de toules natures sur les eaux ot notammont lours droils à l'arrosage. Lo service hydraulique ne saurail d'anllours, dans les curconstances, encourir le reproche d'empiéter sur lo domanne judiciarre, en départageant des intérêts privés : les interrêls agricoles of industriels de toute la partic du cours d'eau dont le régime naturel est complètement modıfié, sonl assez nombreux et assez importantsi pour que leur protection ait nettement le caractère " d'utılité générale "qui, d'après les termes mêmes de la loi des 12-20 août 1790 , iustıfle lintervenlion de l'Administration.

Les dispositions du deuxième paragraphe de l'article 11 ont pour objet d'obliger les usager's des usines ou des prises d'eau établies dans los condilions qui viennent d'être undiquées à transmettre les eaux de façon à ne pas nujire à leur utilisation. Mais ces prescriptions d'ordre général, et en quelque sorte de principe, devront nécessairement être complétées par les obligations spécıales ru'il conviendra d'imposer cn vue du même objed dans l'acte d'autorisation particulier à chaque espèce.

La circulaire qui commentera le décret du $1^{\text {er }}$ août 1905 , donnera, des instructions en ce qui concerne lest clauses de colle nature à insérer" dans les règlements d'eau. Dò̀s maintomant, il y a lieu de veiller avec som à ce que des usines ou des prises d'eau de cette importance no soient jamais, à raison de lcur" influénce sur" le régime des eaux, entreprisus sans autorisation préalable.

AnT. 12. - Déversements interdits. - L'article 12 a pour but d'mlerdire diverses opérations qui pourraient avoir, soit directement, soil indirectement, une influence nuisible sur les cours d'eau.

Le premier parag"raphe vise les dépôts et, d'unei manière générale, les déversements, quels qu'ils suient, qui pourraient gêner l'écoulement des caux.

Le deuxième paragraphie est destuné à protéger la salubrils publique.

Lei trossième paragraphe a pour but d'empêch́s que la qualité des eaux ne soit allérée et que leur" nature ou leur" température ne soit pas modiffées de façon à rendre leur utllis $s_{\lambda}$. tion impossible.

On ne saurait trop insister sur l'importance des prescriptıons des deux derniers paragraphes de l'article 12.

Il a été constaté, à diverses reprises, dans l'exa men des affaires soumises au Ministre de l'Agriculture, qu'un assez grand nombre d'Ingénieurs du servicer hydraulique supposent que le ròle de l'Administration doit se borner à prescrire des mesures en vue d'assurer le libre écoulement des eaux. Cette opinion constitue une grave erreur, puisqu'elle néglige unet partie très importante des attributions de police confér'ées de tous temps à l'autorité administrative et rappelée expressément par l'article 8 de la loi du 8 avril 1898, celle qui est relative à la conservation des cours d'eau.

Limportance de la mission qui incombe à cet égard au service' hydraulique est, d'ailleur's capitale: s'il est nécessaire de prévenir les mondations, il n'est pas moins indispensable, dans l'intérêt général, d'mlerđire que las eaux soient polluées de façon à nurre à la salubrité publıque, de veiller à ce qu'elles ne perdent pás leurs qualités naturelle et d'empê. cher qu'ellos ne solemt r'endues impropres à l'un quelconque des nombreux usages auxquels elles sont destinées.

C'est aux Ingénieurs du service hydraulıque seuls qu'll appartient de proposer aux préfets les mesures qu'il conviendra de prendre afin de protéger" l'alımentation des hommes el des animaux et afin de per'metire l'emploi de l'eau aux usages domestiques ou son utilisalion pour l'agriculture et l'industrie.

En partıculier, loutes les fors qu'il sera utile de compléter les disposilion édıctées par le règlement de polıce, notamment de prévolr cucls sonl les résidus midustrielsı dont l'écouloment est interdit on nee peut être effectué que dans des conditions détermmnées, les Ing'éneur's du service hydraulique devront adresser toutes les proposilnons utiles pour sauvegarder les divers intérêts dont ils onl la charge.

Lorsqu'il ne s'agira que d'assurer' uniquement la conservation du poisson, les Préfels auront à consulter lesı agents de l'Armmistration des For'êts dont dépend lo scrvice de la pêche, mais, on vertu du principe rappelé par la circulairo du 10 décembre 1905, ll conviendra que les mesures qui leur seront soumıses à cet effel fassenl l'objet de conférences avec le service hydrauligue, si elles sond de nature à avoir" une influence sur l'ulilisation industrielle ou agricole des eaux.

En cas de désaccord cntre les conférents lc Mmistre devra èlre saisı sous le timbre de la Direction de l'Hydraulique el des Améliorations agricoles.

Enfin les égouts qui ont mantestement pour objet des déversements rentrant dans la catégorme de ceux qui sont interdits ne peuvent, en principe, otre cxécutés qu'en vertu d'une déclaration d'ulilité publique et clans les condilions reconnucs nécessaires par le service hydraulique pour sauvegarder les divers intérêts qui lui sont confiés, et dont l'importance a été rapinelée.

Cependanl, lorsque ces déversements ne seront pas, par leur composition et leur volume, de nalure à influer à une époque quelconque do l'année, d'une manıère sensible, sur la qualibé des eaux des cours d'éau où lis sont effechués, ils pourronl être assumilés aux écoulements d'caux propres et réglementés dans les mèmes conditions. Maus lor'squ'une ville fera application du système du tont-à-l'égout, l'évacuation dans la rivière devra être déclarée d'utilité publique. Il sera donné d'ailleurs aux Préfets, au sujet des conditions dans lesquolles les égouts peuvent êlre établis, des indications plus détaillées, en leur" adressant, comme le prévoit la circulaire du 22 novembre 1905 , des instructions com plètes sur l'applicalion du déeret du $1^{\text {er }}$ août 1905.

Dispositions parliculières et locales. - I la suite de l'arlicle 12, un blanc est réservé dans ler moldèle du règlement de polıce poum l'insertion des dispostıons particulières et locales qu'il pourrait convenir d'édicter.

S'il cxiste dans le déparlement des parties de rivières non navigables ni flottables dans les vallées desquelles la loi du 28 mai 1858 ait inlertht la construction des ärgues sans auto risalion de l'Administration, il y aura lieu de faire figurer dans cette partie du règlement de pollce l'article suivant :

Conformément à la loi du 28 mai 1858, il ne pourra être établi aucune digue dans les parties submersibles des val. lées des rivières de... (indiquer le nom de ces rivières) sans que les dispositions proịetées aient été, au préalable, soumi. es à l'Administration qui aura le droit d'y apporter toutes les modifications qu'elle iuquera utiles ou même interdire leur exécution. Toute infraction cu.x prescriptions précédentes constitue une contravention de grande voirie dont la connais. sance appartient au Conseil de Préfecture.

Garde-rivières. - L'article qui suit les dispositions particulières et locales est relatif aux garde-rivières. Ces agents 
pourront être instituéls si l'ensemble des intér'essés ou seulement un, certiain nombre d'entre eux ont pris l'engagement solidaire d'assurer les payement de leur traitement; le département et les communes pourront d'ailleurs, s'ils le jugent convenable, accorder des subventions pour aider les intéressés à faire face à cette dépense.

Si le Préfet estime qu'il serait utile dans lintérêt génntal d'habiliter certains agcnts du service hydraulique a iresser des procès-verbaux, il fera connaitre au Ministre 'e livmbro des conducteur's, commis ou cantonniers qu'll croir'a devoir être commissionnés.

Répression des contraventions. - L'avant-dernier article du règlement dé police concerne la répression des contraventions.

Les diverses prescriptions relatives à la constatdition des contravention ${ }_{S}$ et à l'affirmation des procès-verbaux ne soulèvent aucune observation spéciale.

En ce qui concerne la juridiction devant être saisie, quelques indications sont nécessaires. Le lribunal compétent est, d'une manıère générale, le tribunal de simple police ; cepen. dant, l'infraction commise peut, dans certains cas, être poursuivie devant le tribunal correctionnel, notamment sl elle rentre dans l'un des cas prévus par les articles 457 du Coide pénal et 15 de Ia loi des 28 septembre-6 actobre 1791 ; si par exemple, un usinier causait des dommages par suite de la hauteur excessive de son déversoir, par suite du défaut ou de l'insuffisance de levée de ses vannes de décharge, s'il transmettait les eaux d'une manilère nuisible ou altérait, soit directement, soit indirectement, leur qualité.

Indépendamment de l'action r'épressive devant les tribunaux de police pour infraction au règlement sur la police dés eaux, des poursuites devant le conseil de Préfecture pourraient être exercées, si les faits délictueux constituaient des contraventions de grande voirie ; en particulier si une inondations causalt des dommagres à des routes nationales, à des canaux de navigation, à des chemins de fer', à des travaux d'endiguement, s'il y avait lieu, enfin, d'appliquer la loi du 28 mai 1858 relative aux digues établies dans les parties submersibles de certaines rivières.

L'Administration nia, d'ailleur's, pas à s'occuper seulement de la répression pénale de la contravention ou du délit commis; les mesures à prendre en vue de faitre cesser les faits qui sont nuisibles à l'intérêt général présentent une utilité au moins aussi grande ; lels instructions détaillées qui suivent éclaireront sur la procédure à suivre à cet égard.

En vertu des pouvoir's de police conférés à l'Administration, notamment par less loils des 12-20 août 1790 et du 8 avril 1898, le Préfet a toujours le droit de mettre en demeure les intéressés d'effectuer les travaux nécessair'es et de les faire exécuter d'office, s'il n'a pas été temu compte de ses prescriptions.

Mais il est préférable, s'il n'y a pas urgence', d'avoir recour's aux tribunaux auxquels est déféré le procès-verbal de contravention et de leur demander, indépendamment de l'amende prévue, d'ordonner la démolition des ouvragess nuisibles par les soins de l'Administration et aux frais des contrevenants.

En cer qui concerne le recouvrement des dépenses faites, la lol relative aux contributions directes et aux taxes assimilées de chaque exer"cice contient un article qui autorise la mise en recouvrement, au profit de l'Etat, des taxes décer. nées pour dépenses faites d'office au compte des riverains et usagers des courisi d'eau dans l'intérêt de la police et de la répartition des eaux. Le Préfet peut donc décerner contre les contrevenants une taxe qui sera recouvrée comme en matière de contributions directes.

Enfin l'atitention est appeléé sur les observationsis suivantes: :

Les particuliers commettent souvent des contraventions de bonne foi, soit par ignorance, soit par fausse interprétation des règlements ; dans ce cas il conviendra, en général, de ne pas faire dreisser procès-verbal de contravention qu'après avolr enjoint aux intéressés de supprimer ou de modifier les ouvrages nuisibles et les avoir avertis, que, faute de satisfaire à ceis prescriptrons dans un délai de..., ils seront poursuivis devant les tribunaux compétents.

D'autre part, les poursuites ne doivent jamais être exer. cées pour départager des intérêts partıculiers en conflit, mais uniquoment dans l'intérêt général ou en faveur d'un ensemble d'intérêts assez nombreux et assez importants pour justifier l'intervention de l'Administration.

Publication et exécution. - Le dernier article prescrit la publication et l'affichage du règlemont de police dans l'étendue du déprartement, son insertion au Bulletin des actes administratıfs de la préfecture et charge l'Ingénieur en chef, les sous-préfets et les mair"es d'en surveiller et d'en asisurer l'exécution.

Un exemplaire du Bulletin des actes administratifs contenant le règlement de police el hrois exemplailus de laffiche devront être adressés à l'Administration de l'Agriculture.

On ne peut douter", que la vulgarisation des dispositions qui précèdent ne rende de très utiles services aux usagers des cour's d'eau non navigables 11 flottables, en les fixant nellement sur les obligations qui leur incombent. Aussi la plus grande publicité doit êtr'e donnée à l'arrêté préfectoral qui doil être pris conformément au modèle ci-après adressé ; les fontıomnarres chargés de co soin verlleront strictement à son exécution.

En adressant ampliation de la présente circulairc aux Ingénjeurs du service hydraulique, lo Ministre appelle à nouveau toute leur altention annsi qua celle des préfets, sur l'importance des diverses recommandations qui y sont faites afin que l'Administration remplisse, dans toute son étendus et d'une manière efflcace, la mission complexe qui lui in. combe d'assurer la police et la conservation des cours d'eau non navigables ni flottables et de diriger les eaux du territoire vers un but d'utilité gémérale.

\section{LA TRACTION ÉLECTRIQUE} sur les lignes industrielles à voie étroite

La facilité avec laquelle on construit actuellement le matériel de traction électrique, et les prix relativement peu élevés de ce matériel, permettent aujourd'hui d'employer utilement l'électricité pour les transports dans les mines et carrières, et dans bon nombre d'usines ou d'entreprises où l'on a recours aux chemins de fer à voie étroite du type a portatif $D$.

On construit actuellement des locomotives électriques dont la puissance atteint jusqu'à 60 et 80 chevaux, roulant sur des voies de 60 et même 50 centimètres de large, et permettant de remorquer des trains de 25 à 40 tonnes.

La tendance actuelle de réduire le plus possible le prix de revient de la main-d'œuvre, et la diffusion de l'énergie électrique dont les lignes arrivent à sillonner toute la région du Sud-Est, concourent également à mettre en faveur ce type de traction, qui offre l'avantage d'une grande élasticité, d'un rendement meilleur, et qui fatigue moins les voles industrielles que les locomotives à vapeur. Il est d'ailleurs certains cas pour lesquels la rareté de l'eau ou des combustibles, ou bien la circulation dans des galeries réclamant l'absence de fumées, rendent à peu près nécessaire ce genre de traction.

Ce sont des raisons de ce genre qui ont décidé la Société des Bauxites de France à employer la traction électrique sur sa ligne du Recoux au Luc (Var). 